\title{
LETTERS
}

\section{Ambra1 regulates autophagy and development of the nervous system}

\author{
Gian Maria Fimia ${ }^{1 \star}$, Anastassia Stoykova ${ }^{2 \star}$, Alessandra Romagnoli ${ }^{1 \star}$, Luigi Giunta $^{3,5}$, Sabrina Di Bartolomeo ${ }^{3,5}$, \\ Roberta Nardacci ${ }^{1}$, Marco Corazzari ${ }^{1}$, Claudia Fuoco ${ }^{3,5}$, Ahmet Ucar ${ }^{2}$, Peter Schwartz ${ }^{6}$, Peter Gruss ${ }^{2}$, \\ Mauro Piacentini ${ }^{1,4}$, Kamal Chowdhury ${ }^{2}$ \& Francesco Cecconi ${ }^{3,5}$
}

Autophagy is a self-degradative process involved both in basal turnover of cellular components and in response to nutrient starvation or organelle damage in a wide range of eukaryotes ${ }^{1-3}$. During autophagy, portions of the cytoplasm are sequestered by double-membraned vesicles called autophagosomes, and are degraded after fusion with lysosomes for subsequent recycling ${ }^{4}$. In vertebrates, this process acts as a pro-survival or pro-death mechanism in different physiological and pathological conditions, such as neurodegeneration and cancer ${ }^{2,5-7}$; however, the roles of autophagy during embryonic development are still largely uncharacterized $^{3}$. Beclin1 (Becn1; coiled-coil, myosin-like BCL2-interacting protein) is a principal regulator in autophagosome formation, and its deficiency results in early embryonic lethality ${ }^{8,9}$. Here we show that Ambral (activating molecule in Beclin1-regulated autophagy), a large, previously unknown protein bearing a WD40 domain at its amino terminus, regulates autophagy and has a crucial role in embryogenesis. We found that Ambral is a positive regulator of the Becn1-dependent programme of autophagy, as revealed by its overexpression and by RNA interference experiments in vitro. Notably, Ambral functional deficiency in mouse embryos leads to severe neural tube defects associated with autophagy impairment, accumulation of ubiquitinated proteins, unbalanced cell proliferation and excessive apoptotic cell death. In addition to identifying a new and essential element regulating the autophagy programme, our results provide in vivo evidence supporting the existence of a complex interplay between autophagy, cell growth and cell death required for neural development in mammals.

Autophagy is a highly regulated mechanism, as demonstrated by the identification of several autophagy-related (Atg) genes in the yeast Saccharomyces cerevisiae ${ }^{10}$. Recent studies have not only demonstrated the evolutionary conservation of Atg function in vertebrates but also highlighted the involvement of the autophagic machinery in many aspects of tissue homeostasis ${ }^{2,3}$. Indeed, autophagy has a protective function against the onset of neurodegeneration ${ }^{5,6}$ and the alteration of cell growth in cancer ${ }^{7,9,11}$. Moreover, a complex interaction between the pathways of autophagy and apoptosis has been reported in several in vitro systems, in which dysregulation of the apoptotic pathway induces changes to the autophagy programme and vice versa ${ }^{12-15}$.

A role of autophagy in the regulation of vertebrate development has long been proposed by studies reporting morphological features of autophagy during embryogenesis ${ }^{3,16-18}$. Targeted mutagenesis of a few Atg genes in mice substantiated this hypothesis. Embryos that contain a mutation to Becn1, the orthologue of yeast Atg6, die early during embryogenesis, exhibiting a developmental delay', whereas Atg5- or Atg7-deficient mice survive embryogenesis but suffer from nutrient and energy insufficiency soon after birth ${ }^{19,20}$. In addition, $\operatorname{Atg} 5^{-1-}$ embryos were shown to have deficient autophagydependent clearance of apoptotic cell corpses during development ${ }^{21}$. However, it is not yet known how autophagy can be regulated during development, and whether vertebrate-specific factors besides the Atg genes have a role in this context.

Using a large-scale mutagenesis approach based on gene trapping in mice $^{22}$, we isolated a novel gene involved in the development of the nervous system. This gene encodes a 1,300-amino-acid-long WD40 protein that is highly conserved in vertebrates (Supplementary Fig. 1). We named this protein Ambral because of its functions, as described below. In embryos homozygous for the gene-trapped allele $\left(\right.$ Ambra ${ }^{\mathrm{gt} / \mathrm{gt}}$ ) we revealed a fusion transcript (trapped gene-lac $Z$ ) and its corresponding protein, whereas the endogenous protein was absent (Fig. 1a, b). Because $\beta$-galactosidase ( $\beta$-gal) activity in a genetrap line mimics the expression of the tagged endogenous gene ${ }^{23}$, we performed Ambral expression analysis after whole-mount $\beta$-gal staining in heterozygous embryos throughout development and in postnatal brain (see Fig. 1c-e and Supplementary Fig. 2). At embryonic day (E)8.5, strong staining was detected throughout the neuroepithelium (Fig. 1c and Supplementary Fig. 2a). At E11.5 (Fig. 1d, e), a robust expression was seen in the ventral-most part of the spinal cord, the encephalic vesicles, the neural retina, the limbs and the dorsal root ganglia. Messenger RNA in situ hybridization using an Ambral probe confirmed the expression pattern (Fig. If and Supplementary Fig. 2b, compared with $\beta$-gal staining in Supplementary Fig. $2 \mathrm{c})$. At later developmental stages, the $\beta$-gal staining became abundant in the entire developing nervous system as well as in other tissues (Supplementary Fig. 2d); strong expression was observed in the cortex, hippocampus and striatum of postnatal brain (Supplementary Fig. 2e-i).

Notably, we found that homozygosity of the Ambral mutation causes embryonic lethality in outbred or inbred genetic backgrounds (Supplementary Table 1). Most Ambra1 $1^{\mathrm{gt} / \mathrm{gt}}$ embryos at stages E10E14.5 exhibit neural tube defects detected as midbrain/hindbrain exencephaly and/or spina bifida (Fig. 1g-j). Histological analysis revealed that, in the embryonic forebrain of $A m b r a 1^{\mathrm{gt} / \mathrm{gt}}$ mice, the vesicles are closed but displaced, the proliferative neuroepithelium shows an extensive overgrowth and the spinal cord is enlarged (Fig. 1k, 1). Next, we performed a detailed analysis of cell death, differentiation and growth in Ambral $1^{\mathrm{gt} / \mathrm{gt}}$ embryos. Excessive

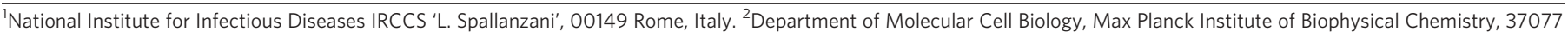
Göttingen, Germany. ${ }^{3}$ Dulbecco Telethon Institute at the Department of Biology, ${ }^{4}$ Department of Biology, University of Rome 'Tor Vergata', 00133 Rome, Italy. ${ }^{5}$ Laboratory of

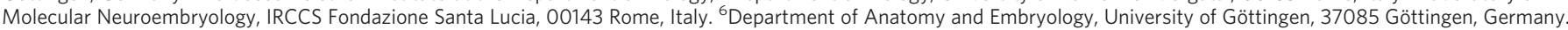
${ }^{\star}$ These authors contributed equally to this work. 
apoptosis was present in selected areas of the mutant nervous system from E9.0 onwards (Fig. $1 \mathrm{~m}-$ p, Supplementary Fig. 3 and data not shown). We then observed that patterning regulators, such as Shh, Fgf8, En1, En2, Gli3, Pax2, Pax3, Pax5, Pax6, Ngn2 (also called Neurog2), Dlx1, Dlx2 and Mash1 (Ascl1), are present at wild-type levels in the developing mutant brain and spinal cord, although a few of them are displaced or show decreased expression (Supplementary Fig. 4 and data not shown). Finally, 5-bromodeoxyuridine (BrdU)uptake experiments and measurement of mitoses revealed a statistically significant increase of proliferating cells in Ambral ${ }^{\mathrm{gt} / \mathrm{gt}} \mathrm{E} 8.5$ neural folds (Fig. 1q, r and Supplementary Fig. 5a, b). However, from E9.5 onwards, the percentage of BrdU-positive cells out of the total number of cells was similar between the mutant and wild-type neuroepithelium, indicating that the hyperproliferation phenotype appears at the onset of neurulation (Fig. 1s, $t$ and Supplementary Fig. 5a). Taken together, these results indicate that Ambra1 is necessary to control cell proliferation and to guarantee cell survival during nervous system development.

To define the biological processes regulated by Ambra1, we identified its molecular interactors using a yeast two-hybrid approach. Complementary DNA encoding the first 667 amino acids of the
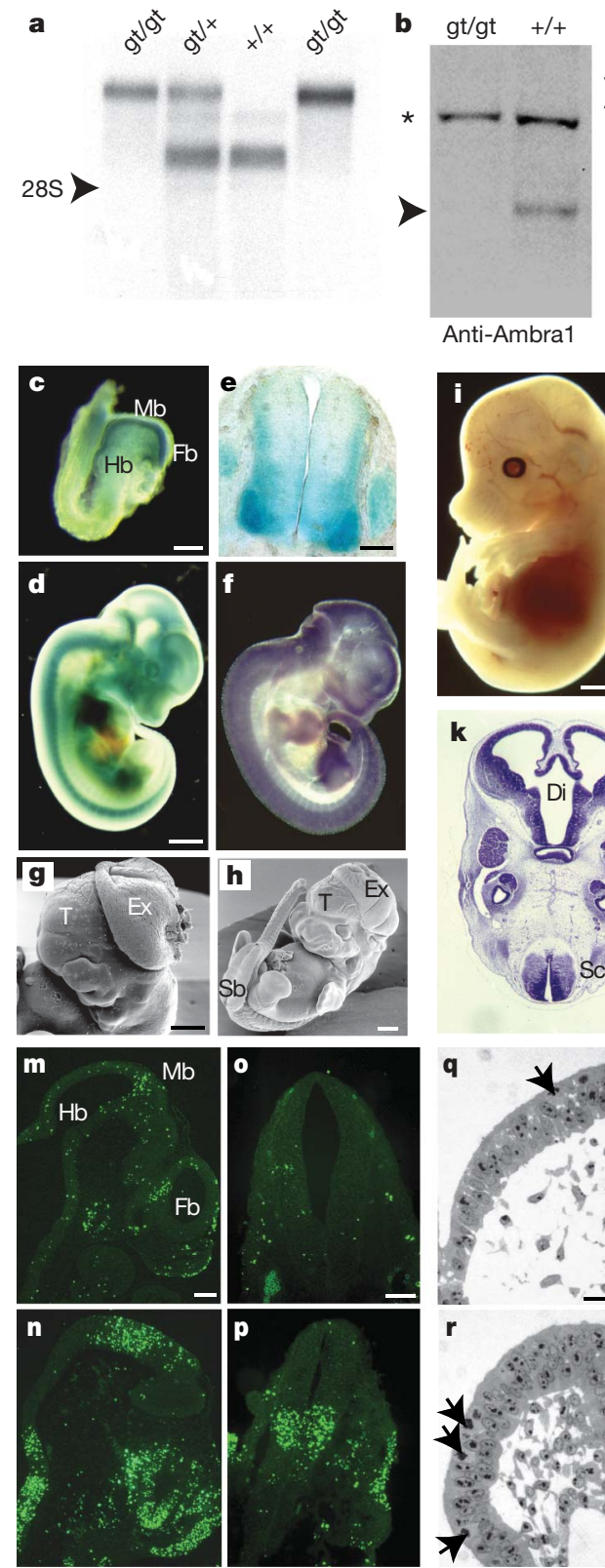
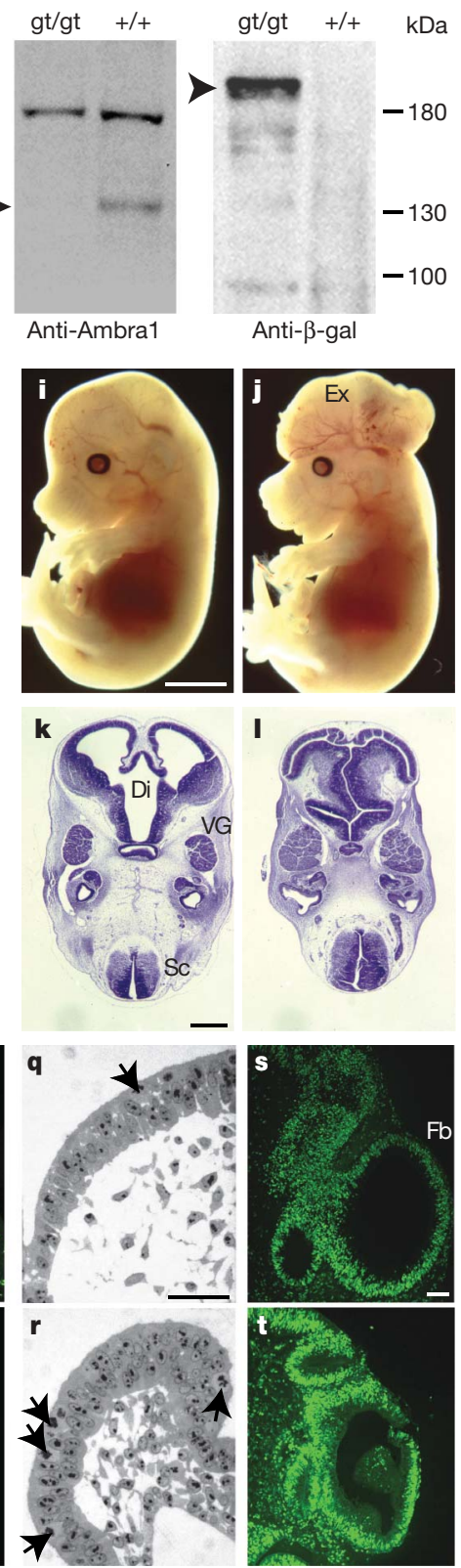

human AMBRA1 orthologue (Supplementary Fig. 1c) was used to screen a human brain cDNA expression library. One of the isolated clones (Fig. 2a) encodes the Atg protein BECN1, a component of the class III phosphatidylinositol-3-OH kinase (also known as Vps34) complex regulating autophagosome formation in mammals ${ }^{8,24}$. The BECN1-AMBRA1 interaction was confirmed in co-transduced human 2FTGH fibroblasts by co-immunoprecipitation assays (Fig. $2 \mathrm{~b}, \mathrm{c})$. To map the region responsible for BECN1 binding, we tested different AMBRA1 mutant constructs in co-immunoprecipitation with BECN1. A central region of the protein (F2) is necessary and sufficient for an effective interaction with BECN1, whereas the AMBRA1 amino terminus (F1) shows a minimal binding capability, and its carboxy terminus (F3) does not interact at all (Fig. 2b). The BECN1-associated kinase Vps34 co-immunoprecipitates with AMBRA1, suggesting that BECN1, Vps34 and AMBRA1 are components of a multiprotein complex (Fig. 2c). The BECN1-AMBRA1 interaction was also confirmed in developing brain samples by coimmunoprecipitating the endogenous proteins (Fig. 2d). Consistent with their interaction, BECN1 and AMBRA1 showed a vesicular-like staining that mostly co-localized in 2FTGH cells (Fig. 2e).

The BECN1-AMBRA1 interaction prompted us to test for a possible role of AMBRA1 in autophagy regulation. The occurrence of autophagy was analysed in 2FTGH cells by assessing both conversion of microtubule-associated protein 1 light-chain 3 (LC3)-I to LC3-II and its translocation to autophagic structures-two sequential steps in autophagosome formation ${ }^{25}$. First we tested the cell response to two autophagic stimuli-the mTOR (mammalian target of rapamycin)-inhibitor rapamycin and nutrient deprivation-upon reduction of AMBRA1 levels by RNA interference. Downregulation of AMBRA1 (Fig. 3a) resulted in a remarkable decrease in autophagy (Fig. 3b, c and Supplementary Fig. 6a). Because BECN1 overexpression has been shown to induce autophagy per $s^{8}$, we tested the effect of AMBRA1 overexpression on autophagy either at basal levels or in rapamycin-treated 2FTGH cells. A significant increase of both basal and rapamycin-induced autophagy was observed (Fig. 3d). Furthermore, we checked whether interaction with BECN1 was required for AMBRA1-induced autophagy by using AMBRA1 F1, F2 and F3 constructs (see Fig. 2b). F2 was able to induce autophagy

Figure 1 Neural tube defects in Ambra1 mutant embryos. a, Detection of wild-type (+) and Ambral gene-trap transcripts (gt) from total E14.5 embryos analysed by northern blotting. Size marker, $28 \mathrm{~S}$ ribosomal RNA. b, Immunoblot analysis of E14.5 embryo brain extracts using antibodies against Ambral (left panel) and $\beta$-gal (right panel). Specific bands (arrowheads) and unspecific signals (asterisk) are indicated. Molecular weights are measured in kilodaltons $(\mathrm{kDa})$. The fusion protein can be revealed only by means of the more sensitive anti- $\beta$-gal antibody. c-f, Expression of Ambral in the mouse embryonic nervous system. $\beta$-galstaining on whole-mount $A m b r a 1^{+/ g t}$ mouse embryos at E8.5 (c), E11.5 (d) and on a cross-section of E11.5 spinal cord (e) is shown. Fb, forebrain; $\mathrm{Hb}$, hindbrain; $\mathrm{Mb}$, midbrain. $\mathbf{f}$, Matching expression of the endogenous gene was revealed by whole-mount Ambral mRNA in situ hybridization analysis in E11.5 wild-type embryos. g-I, Electron scanning microscopy analysis of E11.5 (g) and E12.5 (h) Ambra1 ${ }^{\text {tt/gt }}$ embryos. Note the failure of the neural tube closure, the extensive midbrain/hindbrain exencephaly (Ex) with a closed telencephalon (T), and the lumbosacral spina bifida (Sb). i, j, E14.5 wild-type (i) and Ambra1 ${ }^{\text {gt/gt }}$ (j) embryos are characterized by prominent exencephaly. $\mathbf{k}, \mathbf{I}$, Histological analysis of E12.5 wild-type (k) and Ambra $1^{\text {gt/gt }}$ (I) embryos on cross-section. Note the absence of a normal ventricular system, the extensive overgrowth of the proliferative neuroepithelium in the diencephalon (Di) and spinal cord ( $\mathrm{Sc}$ ), and the enlarged fifth ganglia (VG) in the Ambral ${ }^{\mathrm{gt} / \mathrm{gt}}$ embryo. m-p, TUNEL (TdTmediated dUTP nick end labelling) staining of E10.5 brain $(\mathbf{m}, \mathbf{n})$ and E9.5 spinal cord $(\mathbf{o}, \mathbf{p})$ in wild-type $(\mathbf{m}, \mathbf{0})$ and $A m b r a 1^{\mathrm{gt} / \mathrm{gt}}(\mathbf{n}, \mathbf{p})$ embryo sections. $\mathbf{q - t}$, Analysis of cell proliferation in wild-type $(\mathbf{q}, \mathbf{s})$ and Ambra1 ${ }^{\mathrm{gt} / \mathrm{gt}}$ $(\mathbf{r}, \mathbf{t})$ embryos on transverse sections of E8.5 cephalic neural folds in prospective hindbrain region (mitoses, arrows in $\mathbf{q}, \mathbf{r}$ ) and on sagittal sections of E10.5 forebrain (BrdU uptake, s, t). Scale bars: c, g, h, k, $500 \mu \mathrm{m}$; d, $1 \mathrm{~mm}$; e, $150 \mu \mathrm{m}$; i, $2 \mathrm{~mm}$; m, s, $400 \mu \mathrm{m}$; o, $78 \mu \mathrm{m}$; and q, $50 \mu \mathrm{m}$. 
a
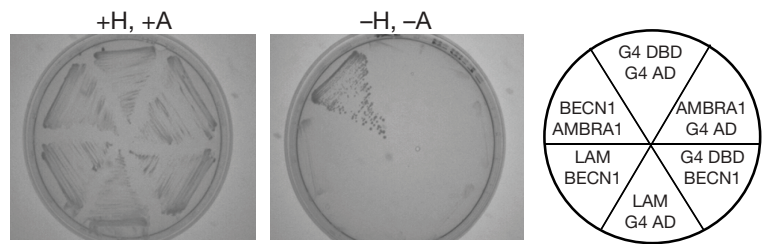

b

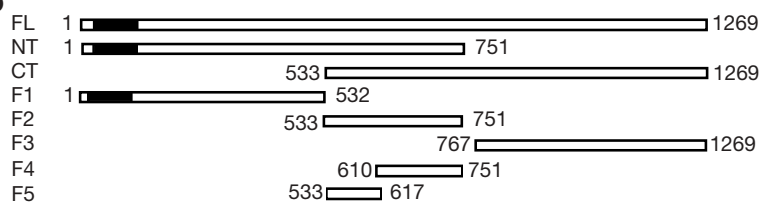

F5
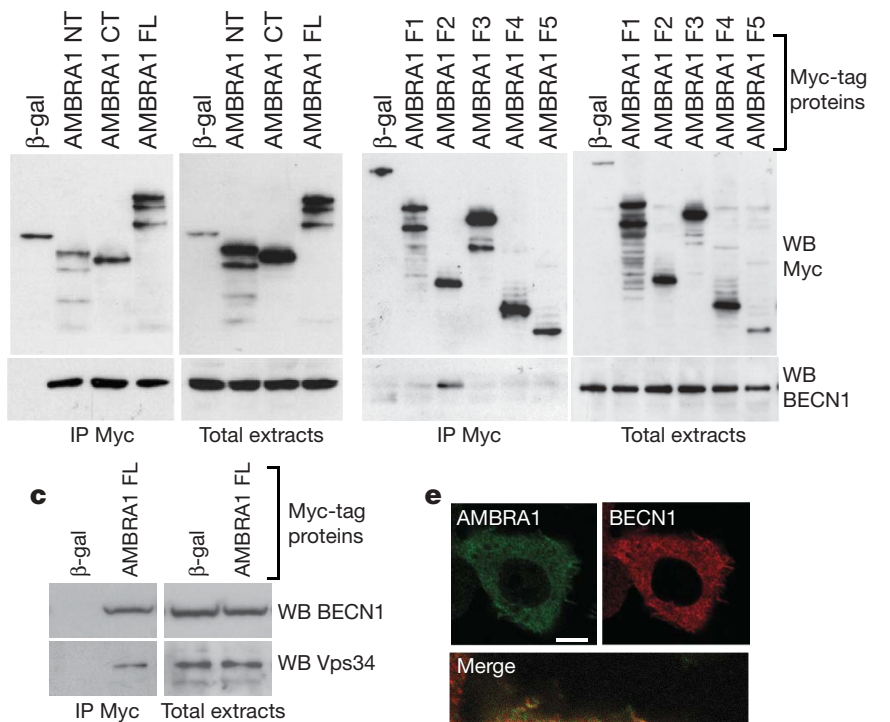

d
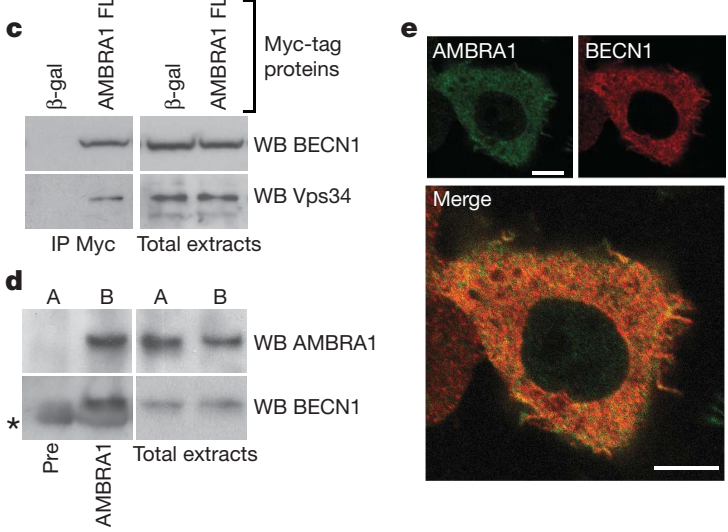

Figure 2 | AMBRA1 is a novel BECN1-interacting protein.

a, AMBRA1-BECN1 interaction by yeast two-hybrid assay. Yeast cells were co-transfected with the indicated plasmids and plated in medium with $(+)$ or without $(-)$ histidine $(\mathrm{H})$ and adenine $(\mathrm{A}) . \mathrm{AD}$, activation domain; $\mathrm{DBD}$, DNA-binding domain; G4, Gal4; LAM, lamin. b, AMBRA1-BECN1 interaction in mammalian cells. 2FTGH cells were co-infected with retroviral vectors encoding BECN1 and the indicated Myc-tagged AMBRA1 proteins, or Myc-tagged $\beta$-gal as a negative control. CT, C terminal; FL, full length; NT, N terminal. Protein extracts were immunoprecipitated using an antiMyc-tagged antibody (IP Myc). Purified complexes and corresponding total extracts were analysed by western blot using anti-Myc (WB Myc, upper panels) or anti-BECN1 (WB BECN1, lower panels) antibodies. When multiple bands are present, the upper band has the expected molecular mass. A scheme of the various AMBRAl mutants is reported. c, AMBRA1 is in a complex that includes BECN1 and Vps34. Protein extracts from 2FTGH cells co-expressing BECN1 and the Myc-tagged AMBRA1 full-length protein (FL) or Myc-tagged $\beta$-gal were immunoprecipitated using an anti-Myc-tag antibody (IP Myc). Purified complexes and corresponding total extracts were analysed using anti-BECN1 (WB BECN1) and anti-Vps34 (WB Vps34) antibodies. d, AMBRA1-BECN1 interaction in developing brain tissues. Protein extracts from two E14.5 wild-type brains (A and B) were immunoprecipitated using an anti-AMBRA1 antibody (AMBRA1) or the pre-immune serum (Pre). Purified complexes and corresponding total extracts were analysed using anti-AMBRA1 (WB AMBRA1) and antiBECN1 (WB BECN1) antibodies. The asterisk indicates the position of comigrating immunoglobulins. e, BECN1 and AMBRA1 co-localize in mammalian cells. 2FTGH cells co-expressing BECN1 and Myc-tagged AMBRA1 were stained for Myc (green) and BECN1 (red). Scale bars, $8 \mu \mathrm{m}$. a
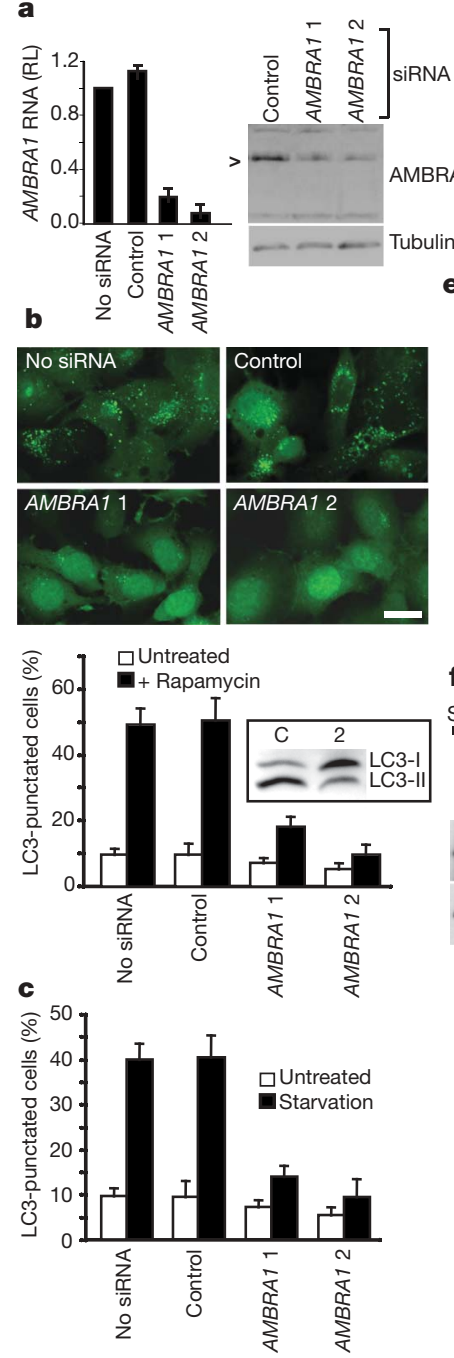

d
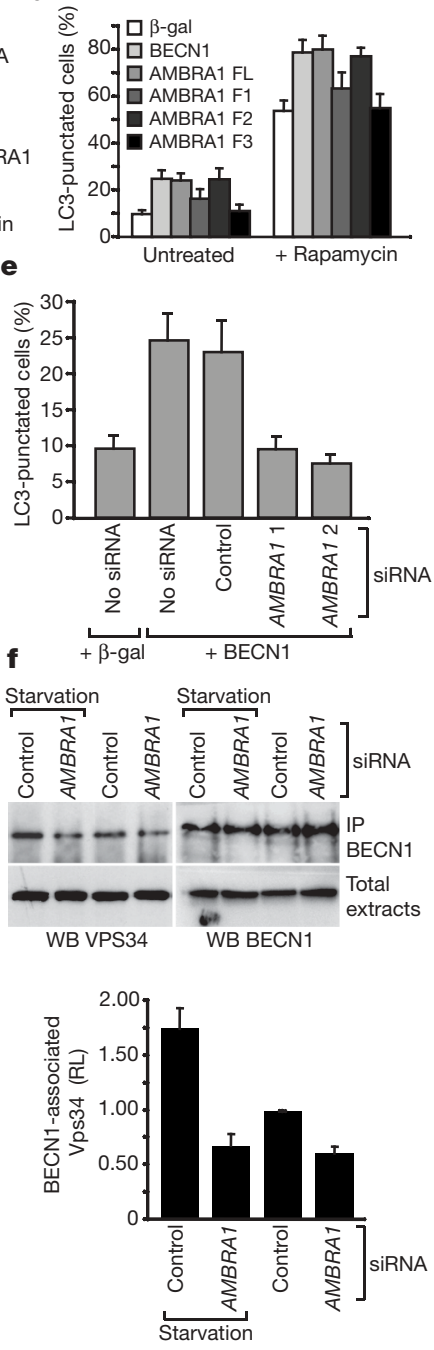

Figure 3 AMBRA1 regulates autophagy. a, AMBRA1 downregulation in 2FTGH cells using specific short interfering RNA (siRNA) oligoribonucleotides (1 and 2). AMBRA1 mRNA and protein levels were analysed by quantitative polymerase chain reaction (PCR, left) and western blotting (right), respectively. An unrelated oligoribonucleotide was used as the control. Tubulin was used as the protein-loading control. RL, relative levels. The arrowhead indicates the AMBRA1-specific band. b, Rapamycininduced autophagy requires AMBRA1. After AMBRA1 downregulation, 2FTGH cells were treated with rapamycin; after $48 \mathrm{~h}$, the occurrence of autophagy was analysed by appearance of GFP-LC3 punctate staining or by LC3-I to LC3-II conversion (western blot in inset). Representative results are accompanied by a graph reporting data from three experiments. Scale bar, $20 \mu \mathrm{m}$. c, Nutrient-starvation-induced autophagy requires AMBRA1. After AMBRA1 downregulation, 2FTGH cells were starved for $4 \mathrm{~h}$ and analysed for appearance of GFP-LC3 punctate staining. d, AMBRA1 overexpression increases basal and rapamycin-induced autophagy. 2FTGH cells were transduced with full-length AMBRA1 (FL), AMBRA1 fragments (F1-3), BECN1 and $\beta$-gal (negative control) encoding retroviruses. They were then stimulated with rapamycin or left untreated, and analysed by appearance of GFP-LC3 punctate staining. e, BECN1-induced autophagy requires AMBRA1. After AMBRA1 downregulation, GFP-LC3-expressing 2FTGH cells were transduced with a BECN1-encoding retrovirus. Occurrence of autophagy was analysed $48 \mathrm{~h}$ later by appearance of GFP-LC3 punctate staining. f, AMBRA1 downregulation reduces the amount of Vps34 associated to BECN1 during autophagy. Forty-eight hours after AMBRA1 downregulation with oligo 2, 2FTGH cells were either starved or cultured in standard medium for an additional $4 \mathrm{~h}$. Protein extracts were

immunoprecipitated (IP) using an anti-BECN1 antibody. Purified complexes and corresponding total extracts were analysed by western blotting with antiVps34 (left panels) and anti-BECN1 (right panels) antibodies. The graph indicates the signal intensity of BECN1-associated Vps34 as determined by densitometry. Values in a-f represent the mean \pm s.d. of three experiments. 
at levels similar to the full-length AMBRA1, F3 had no detectable effect (Fig. 3d), whereas F1 showed an intermediate effect, probably because of its residual BECN1-binding capability. The effects of AMBRA1 dysregulation on the autophagy process were confirmed by measurement of the formation of acidic vesicular organelles ${ }^{26}$ (Supplementary Fig. 6b, c). Also we observed that BECN1-mediated induction of autophagy was markedly reduced after AMBRA1 downregulation (Fig. 3e). Consistently, we showed that AMBRA1 downregulation leads to a reduced capability of BECN1 to interact with its associated kinase Vps34, and to a decrease in Vps34 activity in cells in which autophagy has been induced (Fig. $3 \mathrm{f}$ and Supplementary Fig. $6 \mathrm{~d}-\mathrm{f})$. This finding suggests a role for AMBRA1 in favouring the BECN1-Vps34 functional interaction. Taken together, these results show that AMBRA1 is a key factor in autophagy regulation and is required for BECN1 activity.

The autophagy-promoting activity of BECN1 has been associated with inhibition of cell proliferation ${ }^{8}$. Given the observed hyperproliferative phenotype in Ambral mutant embryos (see Fig. 1q-t and Supplementary Fig. 5), we investigated whether AMBRA1 dosage is critical for cell growth control in vitro. AMBRA1 downregulation or its overexpression resulted in a significant increase or decrease of cell proliferation rate, respectively (Supplementary Fig. 7a, b). Moreover, inhibition of proliferation by AMBRA1 is dependent on BECN1, as this effect is abolished when BECN1 is downregulated (Supplementary Fig. 7c). These observations support the view that dysregulation of BECN1-dependent autophagy is linked to abnormal cell proliferation.

On the basis of these in vitro results, we investigated whether autophagy defects were detectable in Ambral mutants. Conversion of LC3-I to LC3-II was markedly reduced in Ambra1 ${ }^{\mathrm{gt} / \mathrm{gt}}$ E14.5 embryos (Fig. 4a, left panel). LC3 translocation was analysed by crossing Ambral ${ }^{+/ g t}$ mice with transgenic mice ubiquitously expressing green fluorescent protein (GFP)-LC3 (ref. 27). Fluorescence microscopy examination of E10.5 neuroepithelium showed clusters of GFP-LC3 dots in wild-type embryos, whereas a diffuse signal was detectable in Ambral ${ }^{\text {gt/gt }}$ littermates (Fig. 4a, right panels). Next, because autophagy impairment in the adult nervous system is accompanied by accumulation of ubiquinated proteins $s^{5,6}$, we checked for ubiquitin expression in developing brains of $A m b r a 1^{\text {gt/gt }}$ embryos. Numerous ubiquitin-positive cells were present in the mutant neuroepithelium, with a predominant nuclear staining (Fig. 4b). Furthermore, autophagy defects were confirmed in Ambral ${ }^{\text {gt/gt }}$ mouse embryonic fibroblasts, as revealed by GFP-LC3 translocation to autophagosomal membranes, ultrastructural analysis and measurement of formation of acidic vesicular organelles (Fig. 4c, d, Supplementary Fig. $8 \mathrm{a}-\mathrm{c}$ ). Finally, a reduction in the activity of the mutant Ambral protein present in the Ambral ${ }^{\text {gt/gt }}$ mice, in terms of the capability to both interact with Becn 1 and induce autophagy, was confirmed by transfection experiments using a construct mimicking the mutant fusion mRNA (Supplementary Fig. 8d, e). Thus, we conclude that the Ambral gene-trap allele carries a loss-of-function mutation that causes a severe autophagy defect in Ambral ${ }^{\mathrm{gt} / \mathrm{gt}}$ embryos.

Here we show that deficiency of Ambra1, a novel Becn 1 partner mainly expressed in the neural tube during development, impairs autophagy and results in severe neural tube defects. Besides the putative role of AMBRA1 in human congenital brain malformation, its expression profile in adult brain compartments, which are affected in patients with neurodegenerative disorders (Supplementary Fig. 2), suggests a role for AMBRA1 in these diseases, as already shown for other autophagy regulators ${ }^{5,6}$.

Morphogenesis of the nervous system is based on a complex interplay between the control of balanced cell proliferation, differentiation and apoptosis ${ }^{28}$. Our finding that the level of ubiquitinated proteins is markedly enhanced in Ambra1 mutant neuroepithelium implies a role for autophagy-dependent protein turnover in the control of neural development. A sensitive balance of degradation of key neurodevelopmental regulators might be hypothesized as having a role in the determination of cell fate and, when altered, may lead perse to the observed neural tube defects. Alternatively, such a phenotype might be the consequence of disturbed cell proliferation and apoptosis, caused by autophagy dysregulation. In fact, Ambral deficiency during embryogenesis leads to excessive cell proliferation at early stages followed by increased apoptosis in the neuroepithelium. Genetic evidence that autophagy controls cell proliferation was provided by experiments investigating dysregulation of Becn 1 and its
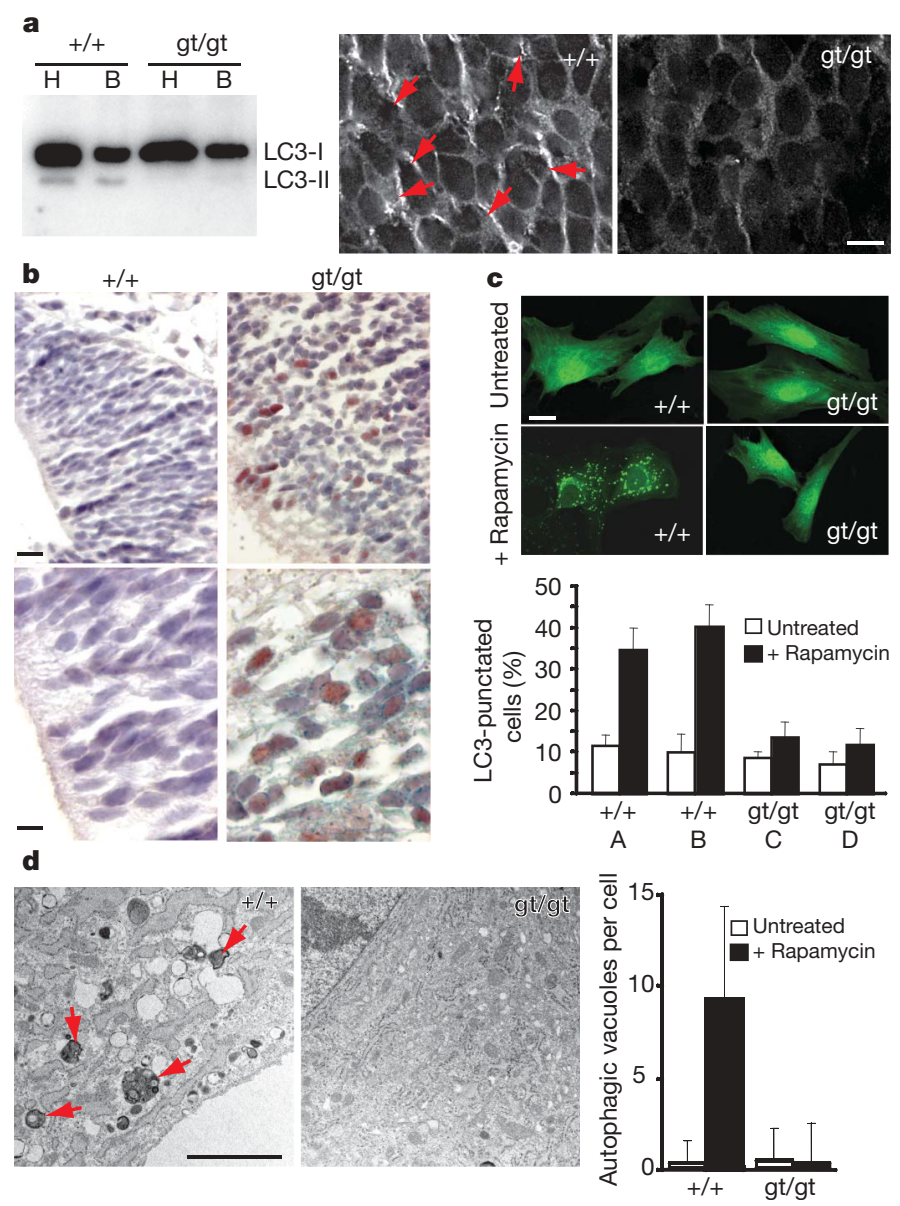

Figure 4 | Autophagy is impaired in Ambra1 mutant embryos. a, Alteration of both LC3 conversion and translocation in Ambral ${ }^{\mathrm{gt} / \mathrm{gt}}$ embryos. Left panel: immunoblot analysis of LC3 in E14.5 embryos. LC3-I to LC3-II conversion is reduced in mutant extracts (LC3-II/LC3-I ratio, as measured by densitometry, from left to right: $5 \%, 9.8 \%, 2.5 \%, 4.6 \%)$. B, body; $\mathrm{H}$, head. Centre and right panels: sections from E10.5 $\mathrm{Ambral}^{+/+}$;GFP-LC3 and Ambra $1^{\text {gt/gt }}$;GFP-LC3 neuroepithelia were compared to evaluate GFP-LC3 subcellular distribution. Clusters of GFP-LC3 punctate structures (arrows) are indicated in wild-type sections. Images were optimized by

deconvolution. Scale bar, $10 \mu \mathrm{m}$. b, Ubiquitin-positive cells in developing brains of Ambra1 ${ }^{\mathrm{gt} / \mathrm{gt}}$ embryos. Sagittal sections from E11.5 embryos were stained using an anti-ubiquitin antibody. Ubiquitin accumulates in numerous mutant neuroepithelial cells (shown at different magnification: upper panels, scale bar, $25 \mu \mathrm{m}$; lower panels, scale bar, $10 \mu \mathrm{m}$ ). Ambral mutant protein is visualized in the left panels by $\beta$-gal activity (light-blue staining). Counterstaining, haematoxylin blue. c, Rapamycin-induced autophagy is impaired in Ambra $1^{\text {gt/gt }}$ murine embryonic fibroblasts (MEFs). Cells dissected from embryos with different genotypes (A-D) were treated with rapamycin, and the occurrence of autophagy was analysed $48 \mathrm{~h}$ later by the appearance of GFP-LC3 punctate staining. Representative results are shown in the upper panels, whereas the lower panel shows the data from three experiments. Scale bar, $20 \mu \mathrm{m}$. d, Ultrastructural analysis by means of electron microscopy of ultrathin sections from wild-type and Ambra $1^{\mathrm{gt} / \mathrm{gt}}$ cultured MEFs. The arrows indicate autophagic vacuoles. Scale bar, $1.5 \mu \mathrm{m}$. Quantification of the mean number of autophagic vacuoles per cell in wildtype and Ambra $1^{\text {gt/gt }}$ cultured MEFs is shown in the graph. Values in $\mathbf{c}$ and d represent the mean \pm s.d. of three experiments. 
interactor ultraviolet radiation resistance associated gene (UVRAG) $8,9,11,29$. Our results suggest that the excessive cell proliferation in the Ambra1 mutant is directly associated with Becn 1 dysregulation. However, we cannot rule out that neuroepithelial cells deficient in autophagy undergo apoptosis in a cell-autonomous fashion, whereas the surrounding cells, lacking proper signalling (for example, Shhmediated mitogenic induction), overproliferate.

The identification of a vertebrate-specific autophagy regulator that is active in an evolutionarily conserved pathway opens up a new scenario involving autophagy in specialized roles during the development of higher eukaryotes.

\section{METHODS SUMMARY}

Generation of Ambral $\mathbf{1}^{\mathrm{gt} / \mathrm{gt}}$ mice. The embryonic stem cell line containing an insertion of the gene-trap vector pGT1.8geo (a gift from W. Skarnes) ${ }^{23}$ within the Ambral gene was generated as described ${ }^{22}$. Founder chimaeric males were mated with outbred NMRI females, and heterozygous progenies were mated to maintain the allele. Also, backcrossing with C57BL/6 males was carried out to obtain an inbred strain. In some experiments, GFP-LC3 mice ${ }^{27}$ were crossed with $\mathrm{Ambral}^{+/ \mathrm{gt}}$ mice to generate Ambral ${ }^{+/ \mathrm{gt}}$;GFP-LC3 mice. The latter were then crossed with each other to obtain Ambral ${ }^{\text {gt/gt }}$;GFP-LC3 embryos.

Autophagy assays. Autophagy was measured in GFP-LC3 transduced cells as described ${ }^{30}$. Briefly, cells grown on coverslips were fixed with $4 \%$ paraformaldehyde in PBS, washed three times and directly examined by confocal microscopy. The results indicate the percentage of GFP-LC3-positive cells with GFP-LC3 punctate dots. A minimum of 50-100 cells per sample were counted for triplicate samples per condition per experiment.

To quantify the development of acidic vesicular organelles ${ }^{26}$, cells were detached by trypsin digestion, washed with PBS, stained with acridine orange ( $1 \mathrm{\mu g} \mathrm{ml}^{-1}$; Sigma-Aldrich) for $15 \mathrm{~min}$ and analysed using a FACScan flow cytometer (Becton Dickinson) and CellQuest software. For electron microscopy, cells were fixed with $2.5 \%$ glutaraldehyde in $0.1 \mathrm{M}$ cacodylate buffer $\mathrm{pH} 7.4$ for $45 \mathrm{~min}$ at $4{ }^{\circ} \mathrm{C}$, rinsed in cacodylate buffer, postfixed in $1 \% \mathrm{OsO}_{4}$ in cacodylate buffer, dehydrated and embedded in Epon. Ultrathin sections were briefly contrasted with uranyl acetate and photographed with a Zeiss EM900 electron microscope.

Full Methods and any associated references are available in the online version of the paper at www.nature.com/nature.

\section{Received 30 January; accepted 2 May 2007.}

Published online 24 June 2007.

1. Lum, J. J., DeBerardinis, R. J. \& Thompson, C. B. Autophagy in metazoans: cell survival in the land of plenty. Nature Rev. Mol. Cell Biol. 6, 439-448 (2005).

2. Levine, B. \& Yuan, J. Autophagy in cell death: an innocent convict? J. Clin. Invest. $115,2679-2688$ (2005).

3. Levine, B. \& Klionsky, D. J. Development by self-digestion: molecular mechanisms and biological functions of autophagy. Dev. Cell 6, 463-477 (2004).

4. Klionsky, D. J. The molecular machinery of autophagy: unanswered questions. J. Cell Sci. 118, 7-18 (2005).

5. Hara, T. et al. Suppression of basal autophagy in neural cells causes neurodegenerative disease in mice. Nature 441, 885-889 (2006)

6. Komatsu, M. et al. Loss of autophagy in the central nervous system causes neurodegeneration in mice. Nature 441, 880-884 (2006).

7. Edinger, A. L. \& Thompson, C. B. Defective autophagy leads to cancer. Cancer Cell 4, 422-424 (2003)

8. Liang, X. H. et al. Induction of autophagy and inhibition of tumorigenesis by beclin 1. Nature 402, 672-676 (1999).

9. Yue, Z., Jin, S., Yang, C., Levine, A. J. \& Heintz, N. Beclin 1, an autophagy gene essential for early embryonic development, is a haploinsufficient tumor suppressor. Proc. Natl Acad. Sci. USA 100, 15077-15082 (2003).

10. Huang, W. P. \& Klionsky, D. J. Autophagy in yeast: a review of the molecular machinery. Cell Struct. Funct. 27, 409-420 (2002).

11. Qu, X. et al. Promotion of tumorigenesis by heterozygous disruption of the beclin 1 autophagy gene. J. Clin. Invest. 112, 1809-1820 (2003).
12. Shimizu, S. et al. Role of Bcl-2 family proteins in a non-apoptotic programmed cell death dependent on autophagy genes. Nature Cell Biol. 6, 1221-1228 (2004).

13. Boya, P. et al. Inhibition of macroautophagy triggers apoptosis. Mol. Cell. Biol. 25, 1025-1040 (2005).

14. Lum, J. J. et al. Growth factor regulation of autophagy and cell survival in the absence of apoptosis. Cell 120, 237-248 (2005).

15. Yousefi, S. et al. Calpain-mediated cleavage of Atg5 switches autophagy to apoptosis. Nature Cell Biol. 8, 1124-1132 (2006).

16. Clarke, P. G. Developmental cell death: morphological diversity and multiple mechanisms. Anat. Embryol. 181, 195-213 (1990).

17. Baehrecke, E. H. How death shapes life during development. Nature Rev. Mol. Cell Biol. 3, 779-787 (2002).

18. Lockshin, R. A. \& Zakeri, Z. Apoptosis, autophagy, and more. Int. J. Biochem. Cell Biol. 36, 2405-2419 (2004).

19. Kuma, A. et al. The role of autophagy during the early neonatal starvation period. Nature 432, 1032-1036 (2004)

20. Komatsu, M. et al. Impairment of starvation-induced and constitutive autophagy in Atg7-deficient mice. J. Cell Biol. 169, 425-434 (2005).

21. Qu, X. et al. Autophagy gene-dependent clearance of apoptotic cells during embryonic development. Cell 128, 931-946 (2007).

22. Stoykova, A., Chowdhury, K., Bonaldo, P., Torres, M. \& Gruss, P. Gene trap expression and mutational analysis for genes involved in the development of the mammalian nervous system. Dev. Dyn. 212, 198-213 (1998).

23. Skarnes, W. C., Moss, J. E., Hurtley, S. M. \& Beddington, R. S. Capturing genes encoding membrane and secreted proteins important for mouse development. Proc. Natl Acad. Sci. USA 92, 6592-6596 (1995).

24. Kihara, A., Kabeya, Y., Ohsumi, Y. \& Yoshimori, T. Beclin-phosphatidylinositol 3-kinase complex functions at the trans-Golgi network. EMBO Rep. 2, 330-335 (2001).

25. Kabeya, Y. et al. LC3, a mammalian homologue of yeast Apg8p, is localized in autophagosome membranes after processing. EMBO J. 19, 5720-5728 (2000).

26. Paglin, S. et al. A novel response of cancer cells to radiation involves autophagy and formation of acidic vesicles. Cancer Res. 61, 439-444 (2001).

27. Mizushima, N., Yamamoto, A., Matsui, M., Yoshimori, T. \& Ohsumi, Y. In vivo analysis of autophagy in response to nutrient starvation using transgenic mice expressing a fluorescent autophagosome marker. Mol. Biol. Cell 15, 1101-1111 (2004).

28. Copp, A. J. Neurulation in the cranial region - normal and abnormal. J. Anat. 207, 623-635 (2005)

29. Liang, C. et al. Autophagic and tumour suppressor activity of a novel Beclin1binding protein UVRAG. Nature Cell Biol. 8, 688-698 (2006).

30. Pattingre, S. et al. Bcl-2 antiapoptotic proteins inhibit Beclin 1-dependent autophagy. Cell 122, 927-939 (2005)

Supplementary Information is linked to the online version of the paper at www.nature.com/nature.

Acknowledgements We thank M. Torres and P. Bonaldo for their involvement in the large-scale gene-trap screening. We also thank A. Conrad for the mouse work, S. Hille for DNA sequencing, M. Acuña Villa and M. W. Bennett for editorial and secretarial work, P. Mattioli for help with image processing, and G. Lisi, and

G. Bellavia G. Marchetti for research assistance. We are grateful to N. Mizushima for providing us with the GFP-LC3 mice. This work was supported in part by grants from the Telethon Foundation and Compagnia di San Paolo (F.C.), Ricerca Corrente and Ricerca Finalizzata from Ministero della Salute and AIRC (M.P.) and the Max Planck Society (A.S., K.C. and P.G.). We thank the Ministry of University and Research of Italy for financial support.

Author Contributions G.M.F., A.R., S.D.B., C.F. and M.C. performed the protein interaction and autophagy assays. A.S. and K.C. generated the Ambra1 gene-trap line and identified the tagged gene. A.S., L.G., K.C., R.N. and S.D.B. carried out the analysis of the phenotype. R.N. and P.S. performed electron microscopy. A.U. generated the Ambra ${ }^{\text {gt }}$ fusion construct. P.G.'s laboratory devised and performed the large-scale gene-trap screening. F.C., M.P. and G.M.F. wrote the paper. All authors discussed the results and commented on the manuscript.

Author Information The sequence for human AMBRA1 cDNA (Supplementary Fig. 1c) has been submitted to GenBank under accession number DQ870924. Reprints and permissions information is available at www.nature.com/reprints. The authors declare no competing financial interests. Correspondence and requests for materials should be addressed to F.C.

(francesco.cecconi@uniroma2.it) or K.C. (kchowdh@gwdg.de). 


\section{METHODS}

Chromosomal localization. For chromosomal localization of the gene-trap insertion site, chromosomal slides were prepared from mouse spleen lymphocytes and hybridized with a biotinylated 2.7-kilobase (kb) cDNA insert as described in ref. 31.

Characterization of the Ambral ${ }^{\text {gt }}$ mutation. Several subsequent rounds of 5' PCR with reverse transcription and cDNA library screenings were performed to obtain the 4,635 base pairs (bp) of compiled cDNA sequence. In detail, after anchored ( $5^{\prime}$ rapid amplification of cDNA ends) PCR (ref. 32), the cDNA template obtained from embryonic stem cell mRNA was amplified by two subsequent rounds of nested PCR. Primers were derived from the lac $Z$ sequence. The amplified products were then blotted and hybridized with a probe specific for the vector splice acceptor site. Once isolated, the corresponding fragment was used as a probe to screen an embryonic mouse cDNA library. After identification of the corresponding cDNA in the databases, the insertion site was localized in the largest 50.36 -kb intron located between the 100- and 113-bp coding exons, as shown in Supplementary Fig. 1b. The vector insertion disrupted the protein at amino acid position 841 (sequence Mus musculus BAE33303.1), thus deleting 459 amino acids from the C-terminal end. For Ambral mRNA analysis, poly $(\mathrm{A})^{+}$RNA was prepared from embryos and $2 \mu \mathrm{g}$ were analysed by standard northern blot analysis.

The construct Ambral ${ }^{\text {gt }}$, encoding the Ambral-LacZ fusion protein present in the gene-trap line, was generated as follows. Briefly, a construct containing the sequences encoding the $\mathrm{N}$-terminal part of the murine Ambral protein as present in the gene-trap line (PCR form cDNA clone IMAGE:5400613) was inserted in-frame within a LacZ-pA (LacZ-polyadenylation site) vector. Next, the Ambral-LacZ-pA fragment $(6.1 \mathrm{~kb})$ was isolated by SmaI-NotI digestion and cloned into the SmaI-NotI cut pCMV Sport 6 vector (German Genome Resource Centre, RZPD) in the sense orientation. To check whether the Ambral ${ }^{\text {gt }}$ construct was able to generate the expected Ambral-LacZ fusion protein in an eukaryotic cell line, we transfected it into the HEK293 cell line using the calcium phosphate method. Cells were fixed and stained for $\beta$-gal activity $24 \mathrm{~h}$ after transfection. Blue staining was already visible after $2 \mathrm{~h}$. Positive and negative control plasmids were also included in the assay, producing the expected results. The full-length murine Ambral clone $\left(A m b r a 1^{\mathrm{wt}}\right)$, used as a positive control, contained the entire coding sequence inserted into the pCMV Sport 6 vector in sense orientation.

Determination of genotype of Ambra1 mutant embryos. To genotype embryos, a Southern blot analysis was performed after digestion of genomic DNA with the BamHI restriction enzyme. The blots were incubated with radioactive probes specific for lac $Z$ and for the housekeeping Fkh5 gene. Densitometric comparison between the two signals was performed to discriminate between heterozygous and homozygous embryos. Early-stage embryos were genotyped by PCR with reverse transcription. Two pairs of primers were used: first, 5'-AACGCATTTATACCCAGTCCA-3' (primer A) and $5^{\prime}$ ACCATAACGTATCGGCCCATC-3' (primer B), mapping upstream and downstream of the gene-trap insertion site, respectively; and, second, primer A together with 5'-CCCAGTCACGACGTTGTAAAA-3' (primer C), the latter one mapping on the lac $Z$ reporter sequence.

Histological and immunocytochemical analyses. Whole-mount $\beta$-gal staining of embryos at different embryonic stages was performed as described ${ }^{33}$. In brief, once removed from the uterus, embryos were washed in PBS and fixed in $4 \%$ paraformaldehyde (PFA) for $30 \mathrm{~min}$ at $4{ }^{\circ} \mathrm{C}$. After washing, they were incubated at $30^{\circ} \mathrm{C}$ in a staining solution containing 5-bromo-4-chloro-3-indolyl- $\beta$-D-galactoside for up to $15 \mathrm{~h}$. The stained embryos were then cut with a vibratome into $60-\mu \mathrm{m}$-thick sections and were examined under a Zeiss Axioplan microscope.

For GFP-LC3 detection of Ambra ${ }^{\text {gt/gt }}$;GFP-LC3 embryos, 10 - $\mu \mathrm{m}$-thick cryostat sections were prepared and analysed under an Olympus IX70 microscope using softWoRx and DeltaVision Imaging Workstation (Applied Precision).

Paraffin sectioning specimens were impregnated with paraffin wax (Paraplast Plus), embedded, transversally sectioned at $10 \mu \mathrm{m}$, and counterstained with cresyl-violet.

For immunohistochemical analysis, the Ambral ${ }^{\text {gt/gt }}$ and wild-type embryos at E9-E9.5 were fixed in 4\% PFA, washed and incubated in $25 \%$ sucrose, before cryo-embedding. Sagittal and coronal $10-\mu \mathrm{m}$-thick sections were blocked in $5 \%$ horse serum and then incubated overnight with the primary antibodies. Fluorescent secondary antibodies (Molecular Probes) were used for signal detection. Cellular nuclei were counterstained with 4,6-diamidino-2-phenylindole or propidium iodide (Molecular Probes). Sections were then examined under an inverted fluorescence microscope (Nikon and Leica). For ubiquitin detection, a biotinylated goat anti-mouse IgG was used as a secondary antibody followed by incubation with horseradish peroxidase-conjugated streptavidin (Biogenex). The immunoreaction product was revealed using 3-amino-9-ethyl-carbazole as a chromogenic substrate and $0.01 \% \mathrm{H}_{2} \mathrm{O}_{2}$ (Biogenex). In cell death experiments, apoptotic cells in embryonic sections were detected by a DNA fragmentation (TUNEL) assay using a fluorescein-based detection kit (Promega). Analysis of cell proliferation was performed after intraperitoneal injection of BrdU (Sigma-Aldrich) using an anti-BrdU antibody (Amersham). For confocal analysis of Becn1-Ambral co-localization and GFP-LC3-transduction experiments, cells were cultured on coverslips and fixed with 4\% PFA in PBS followed by permeabilization with $0.1 \%$ Triton X-100 in PBS. Primary antibodies were incubated for $1 \mathrm{~h}$ at room temperature and visualized by means of Cy3- or Cy2conjugated secondary antibodies (Jackson ImmunoResearch). Coverslips were mounted in Vectashield (Vector Laboratories) and examined under a confocal microscope (Leica TCS SP2).

Antibodies. The primary antibodies used in this study were: rabbit polyclonal anti-Myc Tag antibody (Upstate Biotechnology), goat polyclonal anti-Becnl (Santa Cruz), mouse monoclonal anti-Becnl (Becton-Dickinson), rabbit polyclonal anti-Vps34 (Invitrogen), rabbit polyclonal anti-phospho-p70S6K (Cell Signaling), mouse monoclonal anti-tubulin (Sigma-Aldrich), rabbit polyclonal anti-LC3 (MBL), mouse monoclonal anti $\beta$-gal (Promega), rabbit polyclonal anti-Shh (Santa Cruz) and mouse monoclonal anti-ubiquitin (1B3, MBL). The antibody against Ambral was raised by immunizing rabbits with a peptide located at the amino-terminal of Ambral protein (WEGKRVELPDSPRSC) (Sigma Genosys) and was affinity-purified using the immunizing peptide.

Yeast two-hybrid screening. pGBKT7-AMBRA1 was generated by cloning the $\mathrm{N}$-terminal region (nucleotides 1-2,001) of AMBRA1 into the EcoRI and BglII sites of pGBKT7 (Clontech). A human brain cDNA library cloned in pACT2 (Clontech) was screened by co-transformation with the pGBKT7-AMBRA1 into the AH109 yeast strain. Positive clones were selected based on their growth on Trp, Leu, Ade and His dropout media (Clontech) containing $5 \mathrm{mM}$ 3 -amino-1,2,4-triazole (Sigma-Aldrich). Recovery of the plasmids and the $\beta$-gal assay was performed following the manufacturer's instructions (Matchmaker Two-Hybrid System Protocol, Clontech).

Cell culture. The human fibrosarcoma 2 FTGH cell line, provided by $\mathrm{S}$. Pellegrini, and the human embryonic kidney HEK293 cells, provided by M. Pando, were cultured in DMEM (Sigma-Aldrich) supplemented with 10\% FCS (Sigma-Aldrich), $2 \mathrm{mM}$ L-glutamine, and $1 \%$ penicillin/streptomycin solution at $37^{\circ} \mathrm{C}$ under $5 \% \mathrm{CO}_{2}$. For autophagy induction, cells were treated with $2 \mu \mathrm{M}$ rapamycin (Sigma-Aldrich) or cultured in Earle's balanced salt solution. MEF primary cells were prepared from E13.5 embryos, cultured in DMEM supplemented with $20 \%$ FCS, and utilized for experiments between the second and the seventh passages.

RNA interference. siRNA oligoribonucleotides corresponding to the human AMBRA1 and BECN1 CDNA sequences were purchased from Dharmacon (AMBRA1) and Invitrogen (BECN1, BECN1 Stealth Select RNAi; HSS112742). AMBRA1 SiRNA 1: 5'-AGAACTGCAAGATCTACAA-3; AMBRA1 siRNA 2: 5'-GGCCTATGGTACTAACAAA-3'.

A total of $2 \times 10^{5}$ cells per well were transfected with 100 pmol siRNA in sixwell plates by Lipofectamine 2,000 (Invitrogen), as indicated by the supplier. Transfection was repeated on two consecutive days to increase transfection efficiency. Twenty-four hours after transfection, cells were digested with trypsin, plated at $5 \times 10^{4}$ cells per well in six-well plates and treated with rapamycin (Sigma-Aldrich). The RNA decrease was checked by real-time PCR and western blot $48 \mathrm{~h}$ after transfection.

Cell proliferation analysis. To measure the percentage of cells entering in $\mathrm{S}$ phase, BrdU was added to a final concentration of $20 \mu \mathrm{M}$ and incubated at $37^{\circ} \mathrm{C}$ for $20 \mathrm{~min}$. Cells were detached using trypsin, washed with PBS and fixed using cold $70 \%$ ethanol. DNA was denaturated by adding $4 \mathrm{~N} \mathrm{HCl}$ for $1 \mathrm{~h}$ at room temperature, followed by neutralization with $0.5 \mathrm{M} \mathrm{Na}_{2} \mathrm{~B}_{4} \mathrm{O}_{7}(\mathrm{pH} 8.5)$. Cells were then incubated with anti-BrdU Alexa Fluor $488\left(0.2 \mu \mathrm{g} \mathrm{ml}^{-1}\right.$, Molecular Probes) in PBS plus $0.5 \%$ Tween 20 for $1 \mathrm{~h}$ at $37^{\circ} \mathrm{C}$. Finally, cells were washed with PBS, resuspended in PBS plus $5 \mu \mathrm{g} \mathrm{ml}^{-1}$ propidium iodide and analysed using a FACScan flow cytometer (Becton Dickinson).

cDNA cloning and retroviral vectors. For retroviral expression, all constructs were cloned in a modified version of PLPCX vector (Clontech) in which the SalI restriction site within the puromycin-resistance gene was mutated by PCR to render the Sall site unique in the multiple cloning site. An additional cytomegalovirus promoter was cloned in front of the $5^{\prime}$ long terminal repeat region to increase viral production ${ }^{34}$.

Human AMBRA1 cDNA (Supplementary Fig. 1c) was obtained by PCR amplification from a human brain cDNA library (Clontech) and cloned in EcoRI and NotI sites of the pLPCX vector. The various pCLPCX-AMBRA1 mutants (NT, CT, F1, F2, F3, F4 and F5) were generated by PCR amplification using specific primers. Myc-tagged AMBRA1 fusion proteins were obtained by inserting five copies of the Myc epitope in the HindIII-EcoRI restriction sites of pLPCX. Human BECN1 cDNA was obtained by PCR amplification from a HeLa 
cDNA library (Clontech) and cloned into the EcoRI and SalI sites of pCLPCX. Human LC3 cDNA was obtained by PCR amplification from a HeLa cDNA library (Clontech) and inserted into the EcoRI and SalI sites of pEGFP/C2 (Clontech). GFP-LC3 fusion cDNA was then excised from the pEGFP plasmid by cutting with NheI and SalI restriction enzymes and inserted into pLPCX digested with EcoRI and SalI, with NheI and EcoRI DNA ends blunted to allow the ligation. pLPCX-lacZ-myc was obtained by digesting the pcDNA 3.1 mycHis/lacZ plasmid (Invitrogen) with HindIII-PmeI and inserted into pLPCX digested with HindIII-NotI (blunted). Human p40(phox)PX cDNA (encoding amino acids 13-140 of human p40phox protein) was obtained by PCR amplification from a HeLa cDNA library (Clontech), inserted into the EcoRI and Sall sites of pEGFP/C2 (Clontech) and transfected into the HEK293 cell line using the calcium phosphate method. The sequences of all PCR-amplified cDNAs were verified by DNA sequencing analysis.

Retrovirus generation and infection. Fifteen micrograms of the retroviral vectors were co-transfected with $5 \mu \mathrm{g}$ of an expression plasmid for the vesicular stomatitis virus $\mathrm{G}$ protein into the cell line $293 \mathrm{gp} / \mathrm{bsr}$ (ref. 35) using the calcium phosphate method. Forty-either hours later, the supernatant containing the retroviral particles was recovered and supplemented with polybrene $\left(4 \mu \mathrm{g} \mathrm{ml}^{-1}\right)$. 2FTGH or MEF cells were infected by incubation with retroviral-containing supernantant for 6-8 h.

Real-time PCR. RNA was prepared with Trizol reagent (Invitrogen). cDNA synthesis was generated using the reverse transcription kit (Promega) according to manufacturer's recommendations. Real-time PCR reactions were performed with the LightCycler (Roche). The LightCycler FastStart DNA Master SYBR Green I (Roche) was used to produce fluorescent-labelled PCR products during repetitive cycling of the amplification reaction. Primer sets for all amplicons were designed using the Primer-Express 1.0 software system. AMBRA1 forward: $5^{\prime}-$ AACCCTCCACTGCGAGTTGA-3'; AMBRA1 reverse: 5'-TCTACCTGTTCCGTGGTTCTCC-3'; L34 forward: 5'-GTCCCGAACCCCTGGTAATAGA-3'; L34 reverse: 5'-GGCCCTGCTGACATGTTTCTT-3' .

The result of the fluorescent PCR was expressed as the threshold cycle $\left(C_{\mathrm{T}}\right)$. The $\Delta C_{\mathrm{T}}$ is the difference between the $C_{\mathrm{T}}$ for a specific mRNA and the $C_{\mathrm{T}}$ for a reference mRNA, L34. To determine relative mRNA levels, 2 was raised to the power of $\Delta \Delta C_{\mathrm{T}}$ (the difference between the $\Delta C_{\mathrm{T}}$ from treated cells and the $C_{\mathrm{T}}$ from untreated cells). L34 mRNA level was used as an internal control because this gene was shown to be stable with cell induction. $\beta$-actin and glyceraldehyde3 -phosphate dehydrogenase levels were used as additional controls to confirm significant decreases.

Immunoprecipitation and western blot assays. In immunoprecipitation experiments, cells or tissues were lysed in HEMG buffer $(25 \mathrm{mM}$ HEPES
(pH 8.0), $100 \mathrm{mM} \mathrm{NaCl}, 0.5 \%$ Nonidet P-40, $0.1 \mathrm{mM}$ EDTA, 10\% glycerol) plus protease and phosphatase inhibitors (protease inhibitor cocktail, $1 \mathrm{mM}$ sodium fluoride, $1 \mathrm{mM}$ sodium orthovanadate, $1 \mathrm{mM}$ sodium molibdate; SigmaAldrich). Lysates $(1-2 \mathrm{mg})$ were then incubated with rotation at $4{ }^{\circ} \mathrm{C}$ for $30 \mathrm{~min}$. After a centrifugation at $4{ }^{\circ} \mathrm{C}$ for $10 \mathrm{~min}$ at $13,000 \mathrm{~g}$ to remove insoluble debris, equal amounts of protein were incubated with $30 \mu \mathrm{l}$ monoclonal anticMyc antibody conjugated with protein A agarose beads (BD Biosciences) with rotation at $4{ }^{\circ} \mathrm{C}$ for $4 \mathrm{~h}$, or with $2 \mu \mathrm{g}$ of BECN1 or AMBRA1 antibodies overnight at $4{ }^{\circ} \mathrm{C}$, followed by $60 \mathrm{~min}$ incubation with $20 \mu \mathrm{l}$ of protein $\mathrm{A} / \mathrm{G}$ sepharose beads (Amersham Bioscience). The beads were finally collected by centrifugation and washed four times with the HEMG buffer. Proteins bound to the beads were eluted with $50 \mu \mathrm{l}$ of SDS-polyacrylamide gel electrophoresis sample buffer and heated to $70^{\circ} \mathrm{C}$ for $10 \mathrm{~min}$.

Proteins were separated on NuPAGE Bis-Tris gel (Invitrogen) and electroblotted onto nitrocellulose membranes (Protran, Schleicher and Schuell). Blots were incubated with primary antibodies in $5 \%$ non-fat dry milk in PBS plus $0.1 \%$ Tween 20 overnight at $4{ }^{\circ} \mathrm{C}$. Detection was achieved using horseradish peroxidase-conjugate secondary antibody (Jackson Laboratory) and visualized with ECL plus (Amersham Bioscience).

Embryos from stage E11-E11.5 to E14-E14.5 were subjected to mechanical lysis in $50 \mathrm{mM}$ Tris $\mathrm{HCl}$ (pH 7.5), $320 \mathrm{mM}$ sucrose, $50 \mathrm{mM} \mathrm{NaCl}, 1 \%$ Triton X-100 and protease inhibitors. Solubilized proteins were quantified by a Biorad protein assay, and denatured by adding a concentrated boiling Laemmli buffer. A total of $30-50 \mu \mathrm{g}$ of protein was analysed by SDS-polyacrylamide gel electrophoresis and immunoblotting.

Statistical analysis. Microsoft Excel was used for statistical analysis. Statistical significance was determined using the Student's $t$-test. A $P$ value of less than or equal to 0.05 was considered significant.

31. Heng, H. H. \& Tsui, L. C. Modes of DAPI banding and simultaneous in situ hybridization. Chromosoma 102, 325-332 (1993).

32. Chowdhury, K., Bonaldo, P., Torres, M., Stoykova, A. \& Gruss, P. Evidence for the stochastic integration of gene trap vectors into the mouse germline. Nucleic Acids Res. 25, 1531-1536 (1997).

33. Stoykova, A., Chowdhury, K., Bonaldo, P., Torres, M. \& Gruss, P. Gene trap expression and mutational analysis for genes involved in the development of the mammalian nervous system. Dev. Dyn. 212, 198-213 (1998).

34. Ranga, U. et al. Cell and viral regulatory elements enhance the expression and function of a human immunodeficiency virus inhibitory gene. J. Virol. 71, 7020-7029 (1997).

35. Somia, N. V. et al. LFG: an anti-apoptotic gene that provides protection from Fas-mediated cell death. Proc. Natl. Acad. Sci. USA 96, 12667-12672 (1999). 